\title{
Transdimensional physics and inflation
}

\author{
Gian F. Giudice, Edward W. Kolb, ${ }^{*}$ and Julien Lesgourgues ${ }^{\dagger}$ \\ Theory Division, CERN, CH-1211 Geneva 23, Switzerland \\ Antonio Riotto \\ INFN, Sezione di Padova, Via Marzolo 8, Padova I-35131, Italy
}

(Received 13 July 2002; published 29 October 2002)

\begin{abstract}
Within the framework of a five-dimensional brane world with a stabilized radion, we compute the cosmological perturbations generated during inflation and show that the perturbations are a powerful tool to probe the physics of extra dimensions. While we find that the power spectrum of scalar perturbations is unchanged, we show that the existence of the fifth dimension is imprinted on the spectrum of gravitational waves generated during inflation. In particular, we find that the tensor perturbations receive a correction proportional to $(H R)^{2}$, where $H$ is the Hubble expansion rate during inflation and $R$ is the size of the extra dimension. We also generalize our findings to the case of several extra dimensions as well as to warped geometries.
\end{abstract}

DOI: $10.1103 /$ PhysRevD.66.083512

PACS number(s): 98.80.Cq, 04.50.+h, 11.10.Kk

\section{INTRODUCTION}

The expanding Universe, especially if it underwent a primordial inflationary phase [1], represents the most powerful probe of small distance scales at our disposal. Present-day astronomical length scales were extremely tiny at early epochs and were sensitive to short-distance physics. This simple observation has recently generated a lot of excitement about the possibility of opening a window on trans-planckian or stringy physics in cosmic microwave background (CMB) anisotropies [2]. Unfortunately, in the absence of a quantum theory of gravity, uncontrollable nonlinear effects may dominate at trans-planckian distances, and the behavior of the cosmological perturbations and crucial related issues such as the definition of the vacuum remain unknown. This makes it difficult to predict on firm grounds the signatures of transplanckian physics on present-day cosmological scales [3].

In this paper we will demonstrate that cosmological perturbations generated during inflation may nevertheless provide a powerful probe of another important aspect of many modern theories of particle physics: the existence of extra dimensions. The presence of extra dimensions is a crucial ingredient in theories explaining the unification of gravity and gauge forces. A typical example is string theory, where more than three spatial dimensions are necessary for the consistency of the theory. It has recently become clear that extra dimensions may be very large and could even be testable in accelerator experiments.

In theories with $n$ compactified extra dimensions with typical radii $R$, the four-dimensional Planck mass, $M_{P}$, is just a derived quantity, while the fundamental scale is the gravitational mass, $M_{*}$, of the $(n+4)$-dimensional theory.

\footnotetext{
*Permanent address: Fermilab Astrophysics Center, Fermi National Accelerator Laboratory, Batavia, Illinois 60510-0500 and Department of Astronomy and Astrophysics, Enrico Fermi Institute, The University of Chicago, Chicago, Illinois 60637-1433.

${ }^{\dagger}$ Permanent address: LAPTH, Chemin de Bellevue, B.P. 110, F-74941 Annecy-Le-Vieux Cedex, France.
}

The mass scale $M_{*}$ is a free parameter and can range from a $\mathrm{TeV}$ to $M_{P}$, with $M_{P}^{2} \sim M_{*}^{n+2} R^{n}$. The size of extra dimensions can range from macroscopic scales down to Planckian distances.

In general there is a large hierarchy between the size of extra dimensions, $R$, and $M_{*}^{-1}$, with $R \gg M_{*}^{-1}$. This means that perturbations that are currently observable on cosmological scales might have been generated at early times on scales much smaller than the size of extra dimensions, but still on scales larger than the fundamental Planck mass so that the $(4+n)$-dimensional Einstein equations should describe gravity and the behavior of the quantum vacuum is more certain. This provides a unique probe of the physics of extra dimensions without the necessity of dealing with unknown effects at energies larger than $M_{*}$. This is particularly relevant in brane-world scenarios where gravity propagates in a higher-dimensional space while our visible Universe is a three-dimensional brane in the bulk of extra dimensions [4].

In this paper we initially assume a five-dimensional world where our visible Universe is a three-dimensional brane located at a given point in the fifth dimension. We consider the simplest possibility that inflation is a brane effect, i.e., it is driven by a scalar field living on our three-dimensional brane, and study the effects of the transdimensional physics on the spectrum of the primordial density perturbations produced during the epoch of inflation.

Our findings indicate that despite the fact that the power spectrum of scalar perturbations remains unchanged, the existence of the fifth dimension is imprinted on the spectrum of gravitational waves generated during inflation. The tensor spectrum receives a correction proportional to $(H R)^{2}$, where $H$ is the Hubble rate during inflation and $R$ is the size of the extra dimension. Generalizing our results to the case of more than one extra dimension and to warped geometries, we show that the numerical coefficient of the correction term depends upon the details of the spacetime geometry of the extra dimensions. In four-dimensional single-field models of inflation there exists a consistency relation relating the amplitude of the scalar perturbations, the amplitude of the ten- 
sor perturbations, and the tensor spectral index. We compute the correction to such a consistency relation from transdimensional physics. Surprisingly enough, we find that at lowest order in the slow roll expansion, the four-dimensional relation is quite robust and does not suffer corrections from extra-dimensional physics, at least not in the cases addressed in this paper.

Some similar conclusions have been reached in Refs. $[5,6]$ for a particular five-dimensional setup in which the expansion law on the brane has a non-standard expression. Instead, we will focus on the case where the radius of the extra dimension is stabilized, leading to an ordinary Friedmann law. So any effect should be attributed to the nontrivial geometry along the extra dimension, rather than any modified cosmology on the brane. However, the formalism used in Ref. [5] has many similarities with ours.

Our paper is organized as follows. In Sec. II we study the five-dimensional background with a stabilized radius. In Sec. III we compute the power spectrum of the tensor modes generated during inflation, while in Sec. IV we calculate the power spectrum of scalar perturbations. Section V is devoted to the consistency relation and Sec. VI to a generalization of our findings to more than one extra dimension and to warped geometries. Finally, in Sec. VII we draw our conclusions. The paper also contains an Appendix where we collect the background and perturbed Einstein equations.

\section{A FIVE-DIMENSIONAL BACKGROUND WITH A STABILIZED RADION}

We consider a framework consisting of a $(3+1)$-dimensional brane embedded in a five-dimensional bulk with a stabilized radius. The coordinate along the extra dimension is taken to be $0 \leqslant y<2 \pi R$ (eventually, one may consider to orbifold the circle by a $Z_{2}$ symmetry that identifies $y$ with $-y+2 \pi R$ obtaining the segment $S^{1} / Z_{2}$ ), and the brane is located at $y=0$ at zeroth order in the perturbations. Latin indices $(i=1,2,3)$ label the ordinary three space dimensions; Greek indices $(\mu=0,1,2,3,5)$ run over time, the three ordinary spatial dimensions, and the extra dimension $\mu=5$ (there is no $\mu=4$ ). The background metric may be taken to be of the form

$$
d s^{2}=n^{2}(t, y) d t^{2}-a^{2}(t, y) \delta_{i j} d x^{i} d x^{j}-d y^{2} .
$$

Through a redefinition of time we can always impose the condition $n(t, 0)=1$ in order to obtain the familiar equations on the brane, where the induced metric is simply $d s^{2}=d t^{2}$ $-a_{0}^{2}(t) \delta_{i j} d x^{i} d x^{j}$ [7]. In the background metric there is no time-dependent $b^{2}$ term multiplying $d y^{2}$ because we assume that the radion is stabilized by some unknown high-energy mechanism, and we are free to set $b^{2}=1$. Then for consistency we must assume that the bulk energy-momentum tensor has a non-vanishing (55) component [8] that accounts for the radion stabilizing mechanism, while for simplicity we take the other components of the bulk energy-momentum tensor to be zero. This situation can be achieved by introducing a potential for the radion in the bulk that vanishes at the minimum and whose mass parameter is much larger than the other relevant mass scales. We will see in the following that our results can be generalized to cases with a non-vanishing bulk cosmological constant and a brane tension (like in the Randall-Sundrum framework [9]).

We suppose that the vacuum energy driving inflation is localized on our three-brane at $y=0$ so that the brane energy-momentum tensor provided by the inflaton branefield $\varphi$ is of the form $T_{\nu}{ }^{\mu}=\delta(y) \operatorname{diag}(\rho,-p,-p,-p, 0)$. This might be considered the simplest higher-dimensional scenario to investigate the effects of extra dimensions on cosmological scales. Of course, one may envisage extensions of our set up, such as assuming that the inflaton field $\varphi$ lives in the bulk made of one or more than one extra dimension, or that the spacetime geometry is warped. We will comment of these generalizations at the end of the paper.

We look for solutions of the background (unperturbed) Einstein equations [10]:

$$
\begin{aligned}
G_{0}{ }^{0}= & \frac{3}{n^{2}}\left(\frac{\dot{a}}{a}\right)^{2}-3\left[\frac{a^{\prime \prime}}{a}+\left(\frac{a^{\prime}}{a}\right)^{2}\right]=M_{*}^{-3} \delta(y) \rho(t), \\
G_{j}{ }^{i}= & \left\{\frac{1}{n^{2}}\left[2 \frac{\ddot{a}}{a}+\frac{\dot{a}}{a}\left(\frac{\dot{a}}{a}-2 \frac{\dot{n}}{n}\right)\right]-2 \frac{a^{\prime \prime}}{a}\right. \\
& \left.-\frac{a^{\prime}}{a}\left(\frac{a^{\prime}}{a}+2 \frac{n^{\prime}}{n}\right)-\frac{n^{\prime \prime}}{n}\right\} \delta_{i j} \\
= & -M_{*}^{-3} \delta(y) p(t) \delta_{i j}, \\
G_{5}{ }^{0}= & \frac{3}{n^{2}}\left(\frac{\dot{a}}{a} \frac{n^{\prime}}{n}-\frac{\dot{a}^{\prime}}{a}\right)=0,
\end{aligned}
$$

where $M_{*}$ is the fundamental gravitational mass, $\rho$ is the energy density on the brane, and $p$ is the pressure on the brane. An overdot denotes derivation with respect to $t$, while a prime superscript denotes differentiation with respect to $y$. The $G_{5}{ }^{5}$ equation accounts for the stabilization of the radion and provides a constraint on $T_{5}{ }^{5}$, not on the metric. Other components vanish at zero order in perturbations. In general, the only solution of Eqs. (2)-(4) such that $n(t, 0)=1$ is easily found to be

$$
\begin{aligned}
& a(t, y)=\dot{a}(t, 0)\left[y^{2}-2 \pi R y+\frac{6 \pi R M_{*}^{3}}{\rho(t)}\right]^{1 / 2}, \\
& n(t, y)=\frac{\dot{a}(t, y)}{\dot{a}(t, 0)} .
\end{aligned}
$$

Note that the solution for the background metric is automatically $Z_{2}$-symmetric. Later, we will assume that this is also the case for metric perturbations.

The expression for $a(t, y)$ leads to the standard Friedmann law on the brane expected with a stabilized radion:

$$
H^{2} \equiv\left[\frac{\dot{a}(t, 0)}{a(t, 0)}\right]^{2}=\frac{1}{2 \pi R M_{*}^{3}} \frac{\rho(t)}{3} .
$$


In general, the presence of matter on the brane will cause a small readjustment of the radion with respect to its equilibrium value in vacuum. This shift generates corrections to the Friedmann law in Eq. (6) which are quadratic in $\rho$. Under our assumption that the radion is stabilized by a bulk potential characterized by a mass much larger than the other relevant energy scales, we can safely neglect these corrections.

From this expansion law we can define the fourdimensional gravitational constant to be $M_{P}{ }^{2} \equiv(8 \pi G)^{-1}$ $\equiv 2 \pi R M_{*}^{3}$. Note that when $\pi R H \geqslant 1$, the solution for the scale factor is singular: it is not consistent to impose radion stability when the size of the extra dimension is larger than the Hubble radius, $H^{-1}$. (If the Hubble radius is interpreted as the causal horizon, this just means that the stabilization mechanism must remain causal.)

The singular nature of the scale factor for $\pi R H \geqslant 1$ is not an issue since we are only interested in the case in which the size of the extra dimension is smaller than the Hubble radius, $\pi R H \leqslant 1$, and the cosmological framework is expected to be almost described by four-dimensional physics (up to the correction factors that we wish to calculate).

Therefore, we assume that the energy density during inflation is smaller than $3 M_{P}^{2} /(\pi R)^{2}$, and that deviations from the standard Friedmann law are suppressed up to this scale.

Matching the discontinuity in the components of the Einstein equations (2) and (3) gives the well known jump conditions for $a^{\prime}$ and $n^{\prime}$ :

$$
\left[\frac{a^{\prime}}{a}\right]_{0}^{2 \pi R}=\frac{1}{3 M_{*}^{3}} \rho,\left[\frac{n^{\prime}}{n}\right]_{0}^{2 \pi R}=-\frac{1}{3 M_{*}^{3}}(2 \rho+3 p),
$$

where, for any function $f$, we define

$$
[f]_{\beta}^{\alpha} \equiv f(\alpha)-f(\beta) .
$$

The restriction of the equation for $G_{5}{ }^{0}$ on the brane yields the usual energy conservation law for a perfect fluid: $\dot{\rho}$ $+3 H(\rho+p)=0$.

When the brane only contains a homogeneous inflaton field $\bar{\varphi}(t)$ with potential $V$, the fluid energy conservation law gives the Klein-Gordon equation: $\ddot{\bar{\varphi}}+3 H \dot{\bar{\varphi}}+\partial V / \partial \bar{\varphi}=0$.

If we assume that the density $\rho$ is constant over time, the scale factor is a separable function of time and $y$, and the brane undergoes de Sitter expansion:

$$
\begin{aligned}
a(t, y) & =a_{0}(t) n(y), \quad a_{0}(t) \propto \exp (H t), \\
n(y) & =\left[H^{2}\left(y^{2}-2 \pi R y\right)+1\right]^{1 / 2} .
\end{aligned}
$$

\section{THE PRIMORDIAL SPECTRUM OF TENSOR PERTURBATIONS}

In this section we compute the present-day power spectrum of tensor modes generated by a primordial period of inflation on our visible brane at $y=0$.

The tensor perturbation of the metric is defined, as usual, in terms of a traceless transverse tensor $h_{i j}$ such that

$$
d s^{2}=n^{2}(t, y) d t^{2}-a^{2}(t, y)\left(\delta_{i j}+h_{i j}\right) d x^{i} d x^{j}-d y^{2} .
$$

One learns from the perturbed Einstein equations that the two degrees of polarization contained in $h_{i j}$ obey the wave equation

$$
\ddot{h}+\left(3 \frac{\dot{a}}{a}-\frac{\dot{n}}{n}\right) \dot{h}-\frac{n^{2}}{a^{2}} \Delta h-n^{2} h^{\prime \prime}-n^{2}\left(3 \frac{a^{\prime}}{a}+\frac{n^{\prime}}{n}\right) h^{\prime}=0,
$$

where $h$ is normalized in such a way that $h^{i j} h_{i j}=h^{2} / 2$.

Note that a free scalar field propagating in the bulk would have the same equation of motion as $h$. In the de Sitter background defined in Eqs. (9), and in a Fourier expansion with respect to the three spatial coordinates $x_{i}$, the equation reads

$$
\ddot{h_{k}}+3 H \dot{h}_{k}+\frac{k^{2}}{a_{0}^{2}} h_{k}-n^{2} h_{k}^{\prime \prime}-4 n^{\prime} n h_{k}^{\prime}=0 .
$$

We see from Eq. (9) that $\left(n^{2}\right)^{\prime}$ is not continuous on the brane, and has a jump

$$
\left[2 n n^{\prime}\right]_{0}^{2 \pi R}=4 \pi R H^{2} .
$$

This implies that $\left(n^{2}\right)^{\prime \prime}$ contains a $\delta$ function:

$$
\left(n^{2}\right)^{\prime \prime}=2 H^{2}[1-2 \pi R \delta(y)] .
$$

The solutions of the mode equation, Eq. (12), are separable in $t$ and $y$, so we can expand $h_{k}$ in a sum of Kaluza-Klein modes:

$$
h_{k}=a_{0}^{-3 / 2} n^{-2} \sum_{p} \chi_{p}(t) g_{p}(y)
$$

where $\chi_{p}(t)$ and $g_{p}(y)$ satisfy the equations

$$
\begin{gathered}
\ddot{\chi}_{p}+\left(\frac{k^{2}}{a_{0}^{2}}-\frac{9}{4} H^{2}+\omega_{p}^{2}\right) \chi_{p}=0, \\
n^{2} g_{p}^{\prime \prime}+\left[-\left(n^{2}\right)^{\prime \prime}+\omega_{p}^{2}\right] g_{p}=0 .
\end{gathered}
$$

In the definition of Eq. (15), the factor $a_{0}^{-3 / 2} n^{-2}$ was introduced just for simplicity so that Eqs. (16) and (17) contain no friction terms. The equation for $g_{p}$ has to two independent solutions, which are given in terms of the Gauss hypergeometric function ${ }_{2} F_{1}$,

$$
\begin{aligned}
g_{p}= & c_{12} F_{1}\left(\frac{-1-b}{4}, \frac{-1+b}{4}, \frac{1}{2},-x^{2}\right) \\
& +c_{2} x_{2} F_{1}\left(\frac{1-b}{4}, \frac{1+b}{4}, \frac{3}{2},-x^{2}\right),
\end{aligned}
$$

where the parameters $b$ and $x$ are defined by

$$
b \equiv \sqrt{9-4 \frac{\omega_{p}^{2}}{H^{2}}}, \quad x \equiv \frac{(y-\pi R) H}{\sqrt{1-(\pi R H)^{2}}} .
$$


The metric continuity condition $g_{p}(0)=g_{p}(2 \pi R)$ eliminates the solution odd with respect to $(y-\pi R)$, and fixes $c_{2}=0$. The constant $c_{1}$ is determined by the normalization condition of $g_{p}$. We will set the wave function normalization condition to be

$$
\int_{0}^{2 \pi R} d y n^{-2}\left|g_{p}\right|^{2}=1
$$

Integrating Eq. (17) in a neighborhood of the brane leads to the jump condition for $g^{\prime}$ :

$$
\left[g_{p}^{\prime}\right]_{0}^{2 \pi R}=4 \pi R H^{2} g_{p}(0) .
$$

This condition is satisfied only for a discrete set of possible values of $\omega_{p}$, determined by the equation [11]

$$
\begin{aligned}
{ }_{2} F_{1}\left(\frac{-1-b}{4}, \frac{-1+b}{4}, \frac{1}{2},-a\right) \\
\quad=\frac{(a+1)\left(b^{2}-1\right)}{8}{ }_{2} F_{1}\left(\frac{3-b}{4}, \frac{3+b}{4}, \frac{3}{2},-a\right),
\end{aligned}
$$

with $a$ given by

$$
a \equiv \frac{(\pi R H)^{2}}{1-(\pi R H)^{2}} .
$$

As far as the time dependence is concerned, the solution of Eq. (16) for $\chi_{p}$ is a Bessel function, and can be normalized to the adiabatic vacuum inside the Hubble radius using as usual the positive frequency condition and the canonical commutation relations. To do so, we start from the fivedimensional action

$$
S=\frac{1}{8} \int d t d y d^{3} x M_{*}^{3} n a^{3}\left(n^{-2} \dot{h}^{2}-a^{-2} \delta^{i j} \partial_{i} h \partial_{j} h-h^{\prime 2}\right) .
$$

Note that the factor of $n a^{3}$ simply comes from the term $\sqrt{-g}$. In Fourier space and with the de Sitter background the action is

$$
S=\frac{1}{8} \int d t d y d^{3} k M_{*}^{3} n^{2} a_{0}^{3}\left[\dot{h}_{k} \dot{h}_{k}^{*}+\frac{k^{2}}{a_{0}^{2}} h_{k} h_{k}^{*}-n^{2} h_{k}^{\prime} h_{k}^{* \prime}\right] .
$$

Following Eq. (15), we can expand each mode along the basis formed by the functions $g_{p}$. We define

$$
\begin{aligned}
I_{m p} & =\int_{0}^{2 \pi R} d y n^{-2} g_{m} g_{p}^{*}, \\
J_{m p} & =\int_{0}^{2 \pi R} d y\left(g_{m}^{\prime}-2 \frac{n^{\prime}}{n} g_{m}\right)\left(g_{p}^{* \prime}-2 \frac{n^{\prime}}{n} g_{p}^{*}\right) .
\end{aligned}
$$

After integration over $y$, the effective action reads

$$
\begin{aligned}
S= & \frac{1}{8} \int d t d^{3} k M_{*}^{3} \sum_{m, p}\left\{\left[\dot{\chi}_{m} \dot{\chi}_{p}^{*}-\frac{3}{2} H\left(\chi_{m} \dot{\chi}_{p}^{*}+\dot{\chi}_{m} \chi_{p}^{*}\right)\right.\right. \\
& \left.\left.+\left(\frac{k^{2}}{a_{0}^{2}}+\frac{9}{4} H^{2}\right) \chi_{m} \chi_{p}^{*}\right] I_{m p}-\chi_{m} \chi_{p}^{*} J_{m p}\right\} .
\end{aligned}
$$

Even without knowing explicitly the expression for the $g_{p}$ 's, we can find $I_{m p}$ and $J_{m p}$. The first intermediate step is to integrate by parts

$$
\int_{0}^{2 \pi R} d y g_{m} g_{p}^{* \prime \prime}=-\int_{0}^{2 \pi R} d y g_{m}^{\prime} g_{p}^{* \prime}+4 \pi R H^{2} g_{m}(0) g_{p}^{*}(0) .
$$

So, the integral on the left-hand side has the Hermitian symmetry. Then, we use Eq. (17) and write

$$
\begin{gathered}
g_{m} g_{p}^{* \prime \prime}+\left[-2 H^{2}[1-2 \pi R \delta(y)]+\omega_{p}^{2}\right] n^{-2} g_{m} g_{p}^{*}=0, \\
g_{m}^{\prime \prime} g_{p}^{*}+\left[-2 H^{2}[1-2 \pi R \delta(y)]+\omega_{m}^{2}\right] n^{-2} g_{m} g_{p}^{*}=0 .
\end{gathered}
$$

We subtract these two equations and integrate over $y$, taking advantage of the previously found symmetry. We are left with

$$
\left(\omega_{p}^{2}-\omega_{m}^{2}\right) \int_{0}^{2 \pi R} d y n^{-2} g_{m} g_{p}^{*}=0 .
$$

So, unless $\omega_{p}^{2}=\omega_{m}^{2}$, the above integral vanishes. Given the wave function normalization condition of Eq. (20), we conclude that $I_{m p}=\delta_{m p}$. We can also integrate by parts

$$
J_{m p}=\int_{0}^{2 \pi R} d y\left[n^{-2}\left(g_{m}^{\prime}-2 \frac{n^{\prime}}{n} g_{m}\right)\right]\left(n^{2} g_{p}^{* \prime}-2 n^{\prime} n g_{p}^{*}\right) .
$$

Using the equation of motion and the jump condition for $g_{p}$, we find $J_{m p}=\omega_{p}^{2} \delta_{m p}$. So, the effective four-dimensional action is diagonal:

$$
\begin{aligned}
S= & \sum_{p} \frac{1}{8} \int d t d k^{3} M_{*}^{3}\left[\dot{\chi}_{p} \dot{\chi}_{p}^{*}+\left(\frac{k^{2}}{a_{0}^{2}}+\frac{9}{4} H^{2}-\omega_{p}^{2}\right) \chi_{p} \chi_{p}^{*}\right. \\
& \left.-\frac{3}{2} H\left(\chi_{p} \dot{\chi}_{p}^{*}+\dot{\chi}_{p} \chi_{p}^{*}\right)\right] .
\end{aligned}
$$

Each Kaluza-Klein mode $\chi_{p}$ has the same action as a free field in four-dimensional de Sitter spacetime, and can be quantized following the standard procedure. Namely, the adiabatic vacuum can be defined in the sub-horizon limit $k / a_{0} \gg H$ in which Minkowski spacetime is asymptotically recovered. Then the canonical commutation relation gives

$$
\hat{p}_{\chi_{p}}=\frac{1}{8} M_{*}^{3} \dot{\chi}_{p}, \quad\left[\hat{\chi}_{p}, \hat{p}_{\chi_{p}}^{\dagger}\right]=i,
$$




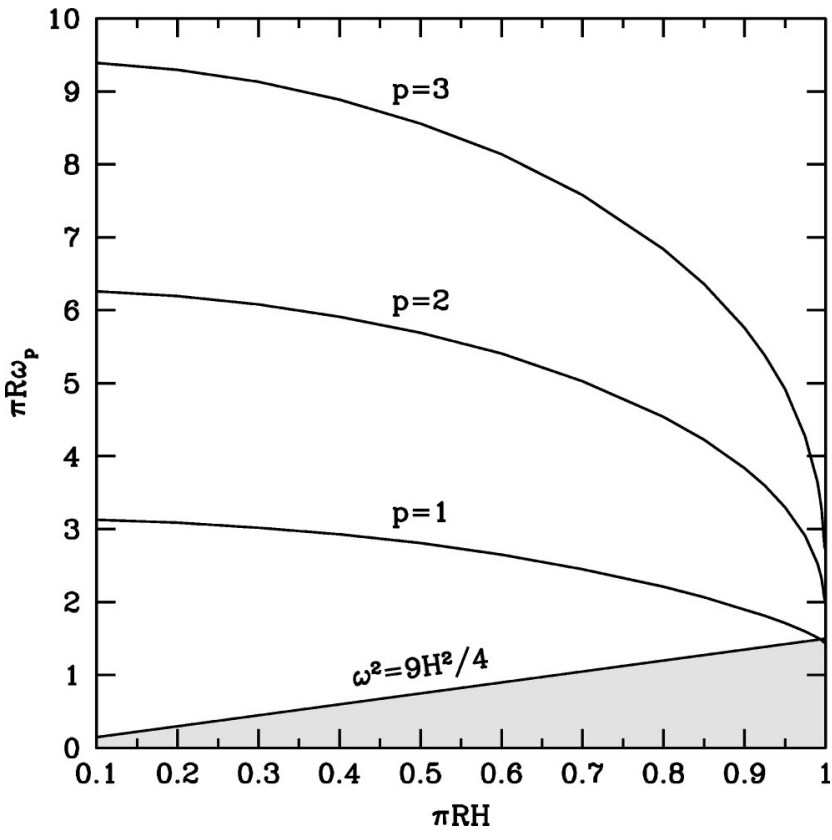

FIG. 1. Effective mass $\omega_{p}$ of the first excited Kaluza-Klein modes for tensor perturbations. For $p=1,2,3$, we plot $\pi R \omega_{p}$ as a function of $\pi R H$. Note that, as expected, as $\pi R H$ approaches zero, $\pi R \omega_{p}$ approaches $p \pi$.

which leads to the Wronskian condition $\chi_{p} \dot{\chi}_{p}^{*}-\dot{\chi}_{p} \chi_{p}^{*}$ $=8 i / M_{*}^{3}$.

Let us focus on the zero mode. Equation (17) with $\omega_{p}$ $=0$ has the obvious solution $c_{0} n^{2}$, where $c_{0}$ is a constant of integration. This solution is automatically continuous and satisfies the jump condition on the brane. The constant of integration is obtained from the condition of Eq. (20):

$$
c_{0}=\left(\int_{0}^{2 \pi R} d y n^{2}\right)^{-1 / 2}=\left[2 \pi R\left(1-\frac{2}{3} \pi^{2} R^{2} H^{2}\right)\right]^{-1 / 2} .
$$

The function $\chi_{0}(t)$ is a Bessel function of index $3 / 2$ and has a simple analytic expression (we retain only the positive frequency solution and we normalize with the Wronskian condition above),

$$
\chi_{0}=\frac{2}{M_{*}^{3 / 2}} \sqrt{\frac{a_{0}}{k}}\left(i \frac{a_{0} H}{k}+1\right) \exp \left(i \frac{k}{a_{0} H}\right) .
$$

After horizon crossing, $\left|\chi_{0}\right|$ grows like $a_{0}^{3 / 2}$. The behavior of the other Kaluza-Klein modes depends on the sign of $\omega_{p}^{2}-9 H^{2} / 4$. As shown in Fig. 1, even $\omega_{1}^{2}$ is larger than $9 H^{2} / 4$ except for a marginal range when $\pi H R$ is very close to 1 . So, all massive Kaluza-Klein modes oscillate at late time with a constant amplitude, and are quickly suppressed with respect to the zero mode by the factor $a_{0}^{3 / 2}$.

In addition, the spectrum of the massive Kaluza-Klein modes is extremely blue, as is usually the case for a scalar field with a mass larger than the Hubble parameter during inflation. This means that for astronomical scales of interest the contribution to the tensor spectrum from Kaluza-Klein modes is practically zero. So, we can focus on the asymptotic value of $h_{k}$ arising from the zero-mode contribution

$$
\begin{aligned}
h_{k}(t, 0) & \rightarrow a_{0}^{-3 / 2} \chi_{0}(t) g_{0}(0) \\
& \rightarrow i\left[2 \pi R M_{*}^{3}\left(1-\frac{2}{3} \pi^{2} R^{2} H^{2}\right)\right]^{-1 / 2} \frac{2}{k^{3 / 2}} H .
\end{aligned}
$$

Even when the de Sitter stage ends, Eq. (11) shows that the zero mode remains frozen on wavelengths larger than the horizon, as is the case in four-dimensional physics. Therefore, the primordial spectrum of gravitational waves at horizon re-entry is still given by Eq. (36), where $H$ has to be evaluated at the time of the first horizon crossing during inflation:

$$
\begin{aligned}
\mathcal{P}_{T}(k) & \equiv \frac{k^{3}}{2 \pi^{2}}\left|h_{k}(y=0)\right|^{2} \\
& =\frac{2}{\pi^{2}}\left(\frac{H(k)}{M_{P}}\right)^{2} \frac{1}{1-2 \pi^{2} R^{2} H^{2}(k) / 3} \\
& =\frac{\mathcal{P}_{T}(k)_{4 D}}{1-2 \pi^{2} R^{2} H^{2}(k) / 3},
\end{aligned}
$$

where $H(k)$ indicates the value of the Hubble parameter when a given wavelength $\lambda=2 \pi / k$ crosses the horizon, i.e., when $k=a_{0} H$. The power spectrum of tensor perturbations is normalized such that in a critical density universe the energy density (per octave) in gravitational waves, $\Omega_{g}(k)$, is related to $\mathcal{P}_{T}(k)$ in terms of the transfer function, $T_{g}^{2}(k)$, by (the transfer function is discussed in Ref. [12])

$$
\Omega_{g}(k)=\frac{1}{24} T_{g}^{2}(k) \mathcal{P}_{T}(k) .
$$

The power spectrum of tensor modes is therefore enhanced compared to the four-dimensional result by a factor (1 $\left.-2 \pi^{2} R^{2} H^{2} / 3\right)^{-1}$.

This correction factor has a simple explanation: it originates from the zero-mode wave function normalization. However, it can be understood also in terms of the effective gravitational Planck mass $\left.M_{P}^{2}\right|_{I}$ during inflation, defined by integrating the zero-mode action over $y$. Indeed, the zero mode, $h_{0} \propto n^{-2} g_{0}$, is constant along $y$ (as expected for a free field with no source localized on the brane). So, integrating the zero-mode action gives a factor (setting $a_{0}=1$ to isolate the gravitational coupling) [13]

$$
\begin{aligned}
\left.M_{P}^{2}\right|_{I} & =M_{*}^{3} \int_{0}^{2 \pi R} d y \sqrt{-g} g^{00} \\
& =M_{*}^{3} \int d y n^{2} \\
& =M_{P}^{2}\left(1-\frac{2}{3} \pi^{2} R^{2} H^{2}\right) .
\end{aligned}
$$


This shows that the enhancement of the tensor power spectrum can be rephrased as a shift in the effective gravitational constant during inflation, when it is defined from the effective gravitational action rather than from the expansion law. The tensor perturbation is a purely five-dimensional field, while the expansion law obtains from a density localized on the brane. This implies that the latter depends on the value of $n^{2}$ on the brane, not on its average. Physically what happens is that the vacuum energy density present on our visible brane during inflation warps the spacetime geometry in the bulk. This effect is manifest in the nontrivial shape of the functions $a^{2}(y)$ and $n^{2}(y)$ during the inflationary epoch. As a result, the graviton zero mode, which is free to spread out in the bulk, feels a smaller Planck mass during inflation. Therefore, today we receive a flux of gravitational waves primordially generated during inflation which is larger than its four-dimensional counterpart because during inflation gravity was stronger.

\section{THE PRIMORDIAL SPECTRUM OF SCALAR PERTURBATIONS}

In this Section we compute the present-day power spectrum of scalar modes generated by a primordial period of inflation on our visible brane at $y=0$.

The first-order scalar perturbations of the metric can be expressed as [14]

$$
\begin{aligned}
d s^{2}= & n^{2}(1+2 \phi) d t^{2}-a^{2}\left[(1-2 \psi) \delta_{i j}+2 \partial_{i} \partial_{j} E\right] d x^{i} d x^{j} \\
& +2 \partial_{i} B d x^{i} d t+2 \partial_{i} w d x^{i} d y+2 \delta g_{05} d t d y \\
& -\left(1-\delta g_{55}\right) d y^{2} .
\end{aligned}
$$

The perturbed brane position is specified by another function, $\delta y\left(t, x^{i}\right)$.

Five-dimensional gauge transformations of the form $x^{\mu}$ $\rightarrow x^{\mu}+\xi^{\mu}$, where $\xi^{\mu}=\left(\xi^{0}, \partial^{i} \xi, \xi^{5}\right)$, induces the transformations

$$
\begin{gathered}
\phi \rightarrow \phi+\dot{\xi}^{0}+\frac{\dot{n}}{n} \xi^{0}+\frac{n^{\prime}}{n} \xi^{5}, \\
\psi \rightarrow \psi-\frac{\dot{a}}{a} \xi^{0}-\frac{a^{\prime}}{a} \xi^{5}, \\
E \rightarrow E+\xi, \\
B \rightarrow B+n^{2} \xi^{0}-a^{2} \dot{\xi}, \\
w \rightarrow w+\xi^{5}+a^{2} \xi^{\prime}, \\
\delta g_{05} \rightarrow \delta g_{05}+\dot{\xi}^{5}-n^{2} \xi^{0 \prime}, \\
\delta g_{55} \rightarrow \delta g_{55}+2 \xi^{5 \prime},
\end{gathered}
$$

while the new brane position is $\delta y\left(t, x^{i}\right)$ $+\xi^{5}\left(t, x^{i}, \delta y\left(t, x^{i}\right)\right)$.

We will work in a particular gauge: the Gaussian normal gauge. The same gauge choice was made in e.g., Ref. [15].
In order to eliminate $\delta g_{55}$, we choose $2 \xi^{5 \prime}=-\delta g_{55}$. This fixes the function $\xi^{5}\left(t, x^{i}, y\right)$ up to a boundary condition, i.e., up to an arbitrary function of $\left(t, x^{i}\right)$ on one hypersurface (for instance, on the brane). The most convenient boundary condition is $\xi^{5}\left(t, x^{i}, \delta y\left(t, x^{i}\right)\right)=-\delta y\left(t, x^{i}\right)$, in order to shift the brane position to $y=0$, even at first order in perturbations. Similarly, in order to eliminate $w$, we may choose $a^{2} \xi^{\prime}=$ $-w-\xi^{5}$, with the boundary condition $\xi=-E$ on the brane in order to have $E=0$ on the brane. Finally, in order to eliminate $\delta g_{05}$, we choose $n^{2} \xi^{0 \prime}=\delta g_{05}+\dot{\xi}^{5}$, with the boundary condition $n^{2} \xi^{0}=-B+a^{2} \dot{\xi}$, so that $B$ also vanishes on the brane. Of course $E$ and $B$ are still non-zero in the bulk. The perturbed metric reduces to

$$
\begin{aligned}
d s^{2}= & n^{2}(1+2 \phi) d t^{2}-a^{2}\left[(1-2 \psi) \delta_{i j}+2 \partial_{i} \partial_{j} E\right] d x^{i} d x^{j} \\
& +2 \partial_{i} B d x^{i} d t-d y^{2} .
\end{aligned}
$$

The induced metric on the brane is diagonal, and involves only the perturbations $\phi_{0}$ and $\psi_{0}$. It is identical to the fourdimensional perturbed metric in the so-called longitudinal gauge. Since the system is symmetric in $y \leftrightarrow 2 \pi R-y$, the perturbations are expected to be even functions with respect to $(y-\pi R)$. In the following, the terms "even" and "odd" will be meant always with respect to $(y-\pi R)$.

We give in Eqs. (A1)-(A5) the expression of the perturbed Einstein equations in the Gaussian normal gauge. In general, the restrictions of the $G_{5}{ }^{0}$ and $G_{5}{ }^{i}$ equations on the brane provide the continuity and Euler equations. When the brane contains only a perturbed scalar field $\varphi\left(t, x^{i}\right)=\bar{\varphi}(t)$ $+\delta \varphi\left(t, x^{i}\right)$, the $\delta G_{5}{ }^{i}$ equation is trivially satisfied, while the $\delta G_{5}{ }^{0}$ equation gives the standard perturbed Klein-Gordon equation:

$$
\delta \ddot{\varphi}+3 \frac{\dot{a}_{0}}{a_{0}} \delta \dot{\varphi}+\left(\frac{\partial^{2} V}{\partial \varphi^{2}}-\frac{\Delta}{a_{0}^{2}}\right) \delta \varphi=\dot{\bar{\varphi}}\left(\dot{\phi}_{0}+3 \dot{\psi}_{0}\right)-2 \frac{\partial V}{\partial \varphi} \phi_{0} .
$$

The other components of the perturbed Einstein equations contain some second derivatives with respect to $y$ that have to be matched with source terms on the brane. However, to first order in the perturbations, the scalar field cannot generate anisotropic stress on the brane: $\delta T_{i j}$ is proportional to $\delta_{i j}$. This imposes the continuity of $E^{\prime \prime}$ across the brane, and therefore, since $E^{\prime}$ is odd, $E_{0}^{\prime}=0$. The other perturbations are sourced on the brane and have to satisfy the jump conditions

$$
\begin{aligned}
-3\left[\psi^{\prime}\right]_{0}^{2 \pi R} & =M_{*}^{-3} \delta T_{0}^{0}=\dot{\bar{\varphi}} \delta \dot{\varphi}-\dot{\bar{\varphi}}^{2} \phi+\frac{\partial V}{\partial \varphi} \delta \varphi \\
-\left[\phi^{\prime}\right]_{0}^{2 \pi R}+2\left[\psi^{\prime}\right]_{0}^{2 \pi R} & =-M_{*}^{-3} \delta T_{i}^{i} \\
& =\dot{\bar{\varphi}} \delta \dot{\varphi}-\dot{\bar{\varphi}}^{2} \phi-\frac{\partial V}{\partial \varphi} \delta \varphi \\
-\frac{1}{2}\left[B^{\prime}\right]_{0}^{2 \pi R} & =M_{*}^{-3} \dot{\bar{\varphi}} \delta \varphi .
\end{aligned}
$$




\section{A. A master equation for the scalar perturbations}

We would like to find an equation of motion for a single variable that would account for the full scalar perturbation dynamics, as Eq. (11) did for tensor perturbations. Such a master equation has already been found in the case of a maximally-symmetric background spacetime [16], but not in cases where $T_{55}$ accounts for the radion stabilization. The best approach is to work with a set of variables reflecting some gauge-invariant quantities. By studying 5-dimensional gauge transformations, it is straightforward to show that one can build four independent gauge-invariant quantities out of the full set of scalar perturbations of the metric. In our gauge, these quantities reduce to

$$
\begin{aligned}
& \Psi_{\phi}=\phi-\frac{\dot{B}}{n^{2}}+a_{0}^{2}\left[-\ddot{E}-2 H \dot{E}+n^{\prime} n E^{\prime}\right], \\
& \Psi_{\psi}=\psi+H \frac{B}{n^{2}}+a_{0}^{2}\left[H \dot{E}-n^{\prime} n E^{\prime}\right], \\
& \Psi_{05}=B^{\prime}-2 \frac{n^{\prime}}{n} B+2 a_{0}^{2} n^{2}\left[\dot{E}^{\prime}+H E^{\prime}\right], \\
& \Psi_{55}=2 a_{0}^{2}\left[n^{2} E^{\prime \prime}+2 n^{\prime} n E^{\prime}\right] .
\end{aligned}
$$

Similarly, it is possible to build a gauge-invariant quantity out of the scalar field perturbation $\delta \varphi$ and the metric perturbations $E$ and $B$. However, in our gauge $E$ and $B$ vanish on the brane, so $\delta \varphi$ directly reflects the gauge-invariant field perturbation. Although the Einstein tensor is not gauge invariant, some of its components can be expressed in terms of ( $\left.\Psi_{\phi}, \Psi_{\psi}, \Psi_{05}, \Psi_{55}\right)$. We write the Einstein equations in the de Sitter background, first in terms of $(\phi, \psi, E, B)$ [see Eqs. (A6)-(A10) of the Appendix], and then in terms of the above variables (some terms in $E$ and $E^{\prime}$ still remain). The traceless part of $\delta G_{j}^{i}$ just gives

$$
\Psi_{\psi}-\Psi_{\phi}-\frac{1}{2} \Psi_{55}=0
$$

and allows us to eliminate easily one of the four variables: instead of $\left(\Psi_{\psi}, \Psi_{\phi}, \Psi_{05}, \Psi_{55}\right)$, we can work with $\left(\Sigma=\Psi_{\psi}\right.$ $\left.+\Psi_{\phi}, \Psi_{05}, \Psi_{55}\right)$. Then, the equation for $\delta G_{0}^{i}$ provides a simple relation between $\Sigma$ and $\Psi_{05}$ :

$$
2 n^{2}(\dot{\Sigma}+H \Sigma)+\left(n^{2} \Psi_{05}\right)^{\prime}=2 M_{*}^{-3} \dot{\bar{\varphi}} \delta \varphi \delta(y) .
$$

The equation for $\delta G_{0}^{0}$, combined with the previous one, gives a relation between $\Sigma$ and $\Psi_{55}$ :

$$
\begin{aligned}
& \frac{3}{2}\left(n^{2} \Sigma^{\prime \prime}+4 n^{\prime} n \Sigma^{\prime}\right)+3 H(H \Sigma+\dot{\Sigma})+\frac{\Delta}{a_{0}^{2}} \Sigma \\
& +\frac{3}{2}\left(\frac{n^{2}}{2} \Psi_{55}^{\prime \prime}+3 n^{\prime} n \Psi_{55}^{\prime}+3 H^{2} \Psi_{55}\right) \\
& =M_{*}^{-3}\left[\delta T_{0}^{0}+6 H \dot{\bar{\varphi}} \delta \varphi+3 H^{2} \Psi_{55}(y=0)\right] \delta(y) .
\end{aligned}
$$

Finally, the system is closed, for instance, by the equation for $\delta G_{5}^{i}$. Indeed, the quantity $a_{0}^{2} n^{-2}\left(n^{4} \delta G_{5}^{i}\right)^{\prime}=0$ can be combined with the previous constraints in Eqs. (47) and (48), to lead to a master equation for $\Sigma$ :

$$
\begin{aligned}
\ddot{\Sigma} & -H \Sigma-\frac{\Delta}{a_{0}^{2}} \Sigma-n^{2} \Sigma^{\prime \prime}-4 n^{\prime} n \Sigma^{\prime}-2 H^{2} \Sigma \\
& =M_{*}^{-3}\left[\delta T_{i}^{i}-\delta T_{0}^{0}+2 a_{0}\left(\frac{\dot{\bar{\varphi}} \delta \varphi}{a_{0}}\right)\right] \delta(y) \\
& =2 M_{*}^{-3}\left(-H \dot{\bar{\varphi}} \delta \varphi+\ddot{\bar{\varphi}} \delta \varphi+\dot{\bar{\varphi}}^{2} \phi_{0}\right) \delta(y) .
\end{aligned}
$$

In the bulk, this equation looks like a five-dimensional wave equation, and in terms of the rescaled variable $a_{0}^{-2} \Sigma$, it would be identical to that of the tensor perturbations or to that of a canonically normalized bulk scalar field. The first difference with the tensor case is the presence of a source localized on the brane, which imposes a jump condition on the derivative $\Sigma^{\prime}$ :

$$
-\frac{1}{2}\left[\Sigma^{\prime}\right]_{0}^{2 \pi R}=M_{*}^{-3}\left(H \dot{\bar{\varphi}} \delta \varphi-\ddot{\bar{\varphi}} \delta \varphi-\dot{\bar{\varphi}}^{2} \phi_{0}\right) .
$$

A similar condition would be found in the case of a bulk scalar field sourced on the brane. However, there is a second difference, reflecting the complicated structure of the Einstein equations, and the integro-differential relations between the various perturbations and boundary conditions. By integrating Eq. (47) over the circle $0 \leqslant y \leqslant 2 \pi R$, we get an integrability condition for $\Sigma$ :

$$
\left\{H+\partial_{t}\right\} \int_{0}^{2 \pi R} d y n^{2} \Sigma=M_{*}^{-3} \dot{\bar{\varphi}} \delta \varphi .
$$

Any even solution of the master equation, Eq. (49), matching the jump and integrability conditions, Eqs. (50) and (51), provides a solution of the full Einstein equations. At any time one can compute the perturbations $\left(\phi_{0}, \psi_{0}\right)$ on the brane. Indeed, the integral of Eq. (48) over $y$ gives $\Psi_{55}(y=0)$ as a function of $\Sigma$ and of the scalar field (remembering that $\left[\Psi_{55}^{\prime}\right]_{0}^{2 \pi R}$ is given by the jump conditions, while $\left.\int_{0}^{2 \pi R} d y \Psi_{55}=2 a_{0}^{2}\left[n^{2} E^{\prime}\right]_{0}^{2 \pi R}=0\right)$ : 


$$
\begin{aligned}
2 \pi R H^{2} \Psi_{55}(y= & 0)=-\left\{\partial_{t}^{2}+H \partial_{t}-\frac{\Delta}{3 a_{0}^{2}}\right\} \int_{0}^{2 \pi R} d y \Sigma \\
& +\frac{2}{3} M_{*}^{-3}\left(\dot{\bar{\varphi}} \delta \dot{\varphi}+2 \ddot{\bar{\varphi}} \delta \varphi+2 \dot{\bar{\varphi}}^{2} \phi_{0}\right) .
\end{aligned}
$$

Finally, the perturbations on the brane may be found from

$$
\begin{aligned}
& \psi_{0}=\frac{1}{2}\left[\Sigma(y=0)+\frac{1}{2} \Psi_{55}(y=0)\right], \\
& \phi_{0}=\frac{1}{2}\left[\Sigma(y=0)-\frac{1}{2} \Psi_{55}(y=0)\right] .
\end{aligned}
$$

\section{B. Comparison to the four-dimensional solution}

In four dimensions, the absence of anisotropic stress implies $\phi_{0}=\psi_{0}$ and scalar metric perturbations are described by a single variable (matching the Newtonian gravitational potential inside the Hubble radius). The exact computation of the scalar power spectrum for scales leaving the Hubble radius during inflation can be performed by various methods that take into account the coupling between the metric and the scalar field perturbations [17]. For instance, one can integrate the equation of propagation of the Mukhanov variable which is a combination of $\delta \varphi$ and $\phi_{0}$ [18]. A second possibility is to solve a pair of coupled equations: the perturbed Klein-Gordon equation (43), and one of the Einstein equations, for instance that for $\delta G_{0}^{i}$ :

$$
\dot{\phi}_{0}+H \phi_{0}=\frac{\dot{\bar{\varphi}} \delta \varphi}{2 M_{P}^{2}} .
$$

In the present work, we are interested in the generalization of the scalar power spectrum calculation only at the leading order in slow-roll parameters. In four dimensions, such a calculation is done consistently by writing all the equations of propagation in the exact de Sitter background; simultaneously, in the expression for the perturbations one keeps the leading-order term in the expansion parameter $\dot{H} / H^{2}$ [or equivalently $\left.\dot{\bar{\varphi}}^{2} /\left(H^{2} M_{P}^{2}\right)\right]$, treated as a constant. In that case, the sourcing of the field perturbation by the metric perturbation [described by the right-hand side in the Klein-Gordon equation Eq. (43)] can be safely neglected, and one can solve the homogeneous equation

$$
\delta \ddot{\varphi}+3 H \delta \dot{\varphi}+\left(\frac{\partial^{2} V}{\partial \varphi^{2}}+\frac{k^{2}}{a_{0}^{2}}\right) \delta \varphi=0 .
$$

At leading order in slow-roll parameters we can also neglect $\left|V^{\prime \prime}\right| \ll H^{2}$ and write the solution, normalized to the adiabatic vacuum inside the Hubble radius, as

$$
\delta \varphi=\frac{1}{a_{0} \sqrt{2 k}}\left(1+i \frac{H a_{0}}{k}\right) \exp \left(i \frac{k}{a_{0} H}\right) .
$$

The metric perturbation is just following the field evolution, according to Eq. (54). This gives in the two limits (subHubble-radius and super-Hubble-radius scales):

$$
\begin{aligned}
& \delta \varphi \simeq \frac{1}{a_{0} \sqrt{2 k}} \exp \left(i \frac{k}{a_{0} H}\right), \\
& \phi_{0}=\frac{i \dot{\bar{\varphi}}}{2 M_{P}^{2} \sqrt{2 k^{3}}} \exp \left(i \frac{k}{a_{0} H}\right) \quad\left(\text { for } \frac{k^{2}}{a_{0}^{2}} \gg H^{2}\right), \\
& \delta \varphi \simeq \frac{H}{\sqrt{2 k^{3}}}, \\
& \phi_{0}=\frac{\dot{\bar{\varphi}}}{2 M_{P}^{2} \sqrt{2 k^{3}}} \quad\left(\text { for } \frac{k^{2}}{a_{0}^{2}} \ll H^{2}\right) .
\end{aligned}
$$

In both limits, we see that the right-hand side in the KleinGordon equation is always very small, and acts as a source for the field perturbations only at a sub-leading order in $\dot{H} / H^{2}$ :

$$
\left|\dot{\bar{\varphi}}\left(\dot{\phi}_{0}+3 \dot{\psi}_{0}\right)-2 \frac{\partial V}{\partial \varphi} \phi_{0}\right| \sim\left|\frac{\dot{\bar{\varphi}}^{2}}{M_{P}^{2}} \delta \varphi\right| \sim|\dot{H} \delta \varphi| \ll H^{2}|\delta \varphi| .
$$

Another way to reach the same conclusion is to look at the Mukhanov variable $\xi=a_{0}\left(\delta \varphi+\dot{\bar{\varphi}} \phi_{0} / H\right)$. It is straightforward to show that at leading order in slow-roll parameters, $\delta \varphi$ and $\xi / a_{0}$ are equal and share the same equations of propagation. The distinction becomes only relevant when slow roll is marginally or temporarily violated, for instance, in inflationary models with phase transitions.

We reviewed this point in the four-dimensional case because it is crucial for the five-dimensional calculation where we will also assume that at leading order $\delta \varphi$ can be treated as a free field, obeying Eq. (55). We will check a posteriori that the metric perturbations just follow the field and do not alter $\delta \varphi$ in a significant way.

\section{Long-wavelength solution in the five-dimensional model}

If the assumption that the scalar metric perturbations just follow the scalar field perturbations is correct, then it is sufficient to study the coupling between these two degrees of freedom in the long-wavelength regime in order to know the power spectrum $k^{3}\left|\phi_{0}\right|^{2}$ on super-Hubble-radius scales during inflation. This is our purpose in this section. In the next section we will study the short-wavelength solution for consistency.

On super-Hubble-radius scales the scalar field perturbations are approximately constant in time: $\delta \varphi=H / \sqrt{2 k^{3}}$ (we dropped the arbitrary phase). We first look for a particular solution of the inhomogeneous master equation, Eq. (49), neglecting the Laplacian term. In slow roll only the first of the three contributions to the source term on the right-hand 
side is relevant because $|\ddot{\bar{\varphi}} \delta \varphi| \ll|H \dot{\bar{\varphi}} \delta \varphi|$ and $\left|\dot{\bar{\varphi}}^{2} \phi_{0}\right|$ $\sim\left|\dot{H} \phi_{0}\right| \ll H^{2}\left|\phi_{0}\right|$. A particular solution is found to be

$$
\Sigma_{i}=\frac{1}{2 \pi R M_{*}^{3}} \frac{\dot{\bar{\varphi}} \delta \varphi}{H n^{2}}
$$

This solution is constant in time, but not in $y$. It matches both the jump and integrability conditions Eqs. (50) and (51) (still at leading order in $\dot{H} / H^{2}$ ). We are free to add to Eq. (60) a solution of the homogeneous equation, i.e., any even solution $\Sigma_{h}$ of the sourceless master equation

$$
\Sigma_{h}-H \Sigma_{h}-n^{2} \Sigma_{h}^{\prime \prime}-4 n^{\prime} n \Sigma_{h}^{\prime}-2 H^{2} \Sigma_{h}=0
$$

such that $\left[\Sigma_{h}^{\prime}\right]_{0}^{2 \pi R}=0$ and

$$
\left\{H+\partial_{t}\right\} \int_{0}^{2 \pi R} d y n^{2} \Sigma_{h}=0
$$

We almost already have the solutions because the homogeneous master equation written in terms of $\left(a_{0}^{-2} \Sigma_{h}\right)$ is the same as the equation for the tensor modes. So $\Sigma_{h}$ is a sum of separable solutions (the Kaluza-Klein modes) with the same values of $\omega_{p}$ as for the tensors, but with a different normalization condition, Eq. (62). For the zero mode with $\omega_{p}=0$, the solution reads $\left(\Sigma_{h}\right)_{p=0}=C_{1} a_{0}^{2}+C_{2} a_{0}^{-1}$, but the normalization condition imposes $C_{1}=0$. For heavy modes with $\omega_{p} \geqslant 3 H / 2$, one gets on super-Hubble-radius scales

$$
\left(\Sigma_{h}\right)_{p}=C_{p} a_{0}^{1 / 2} \exp \left( \pm i t \sqrt{\omega_{p}^{2}-\frac{9}{4} H^{2}}\right) n^{-2}(y) g_{p}(y),
$$

where $g_{p}$ is an even solution of Eq. (17). But, since the integral of $g_{p}$ over $y$ does not vanish, the integrability condition imposes $C_{p}=0$ : in the limit under consideration, the Einstein equations are not compatible with any significant contribution of heavy Kaluza-Klein modes. The final solution reads

$$
\Sigma=\frac{1}{2 \pi R M_{*}^{3}} \frac{\dot{\bar{\varphi}} \delta \varphi}{H n^{2}}+\frac{C_{2}}{a_{0}}
$$

and corresponds to the usual combination of a growing adiabatic mode driven by the scalar field, and a decaying mode that could be normalized only if the full solution was known (from inside the horizon). Since the decaying mode becomes rapidly negligible it will not concern us. Then, we can compute $\Psi_{55}(y=0)$ using Eq. (52). The leading-order terms are

$$
\begin{aligned}
& 2 \pi R H^{2} \Psi_{55}(y=0) \\
& \quad \simeq-H \partial_{t} \int_{0}^{2 \pi R} d y \Sigma+\frac{2}{3} M_{*}^{-3} \dot{\bar{\varphi}} \delta \dot{\varphi} \\
& \\
& \simeq-\frac{2}{3} \pi R \dot{H} \Sigma(y=0)\left[1+\mathcal{O}\left(H^{2} R^{2}\right)\right] .
\end{aligned}
$$

So, to first order in $\epsilon=-\dot{H} / H^{2}=M_{P}^{2}\left(V^{\prime} / V\right)^{2} / 2$, we recover $\psi_{0}=\phi_{0}=\Sigma_{0} / 2$, and the metric perturbations on the brane match exactly the four-dimensional result:

$$
\phi_{0}=\psi_{0}=\frac{1}{4 \pi R M_{*}^{3}} \frac{\dot{\bar{\varphi}}}{H} \delta \varphi=\frac{\dot{\bar{\varphi}}}{2 M_{P}^{2} \sqrt{2 k^{3}}} .
$$

Since our results indicate that on long wavelengths the perturbations of the gravitational potential coincides with the four-dimensional one, the power spectrum of scalar curvature perturbations will be given by the usual result (we recall that during the de Sitter stage, the large wavelength metric perturbations are related to the curvature perturbations by a factor $\epsilon$ )

$$
\mathcal{P}_{S}(k)=\frac{k^{3}}{2 \pi^{2}} \frac{\phi_{0}^{2}}{\epsilon^{2}}=\frac{1}{8 \pi^{2}} \frac{1}{\epsilon}\left(\frac{H(k)}{M_{P}}\right)^{2}
$$

Here $\mathcal{P}_{S}(k)$ is defined in terms of the observable power spectrum $P(k)$ and the scalar transfer function $T^{2}(k)$ by

$$
\frac{k^{3}}{2 \pi^{2}} P(k)=\left(\frac{k}{a H}\right)^{4} T^{2}(k) \mathcal{P}_{S}(k) .
$$

In Eq. (67), the limit $\epsilon=0$ is singular as in four dimensions. This corresponds to the exact de Sitter limiting case, for which $\dot{\bar{\varphi}}=\ddot{\bar{\varphi}}=0$. Then, the master equation Eq. (49) and the constraint equations Eqs. (50), (51) have vanishing righthand sides. The single solution for $\Sigma$ at large wavelength is the decaying mode, $C_{2} / a_{0}$ found in Eq. (64). Plugging this mode into Eq. (52) shows that $\Psi_{55}(y=0)=0$ and $\phi_{0}=\psi_{0}$ $=C_{2} / 2 a_{0}$. We reach the same conclusion as in four dimensions: for exact de Sitter expansion, the scalar metric perturbations do not have a non-decaying solution at large wavelength.

It is appropriate to re-emphasize our result that the scalar spectrum is unaltered is only true to lowest order in the slowroll parameters. For instance, one sees that Eq. (65) has corrections of order $\left(H^{2} R^{2}\right)$, but they are multiplied by $\epsilon$, so to lowest order in the slow-roll parameters we can ignore them.

The absence of any correction factor in $(R H)^{2}$ can be interpreted in the following way: Unlike the tensor degrees of freedom, which are five-dimensional free fields quantized in the bulk, the scalar metric perturbations only follow the scalar field. The later is quantized on the brane and has the same behavior as in the four-dimensional case in all regimes. So we only need to study the coupling between the field and the metric in the long-wavelength regime when the metric evolves as in the four-dimensional theory. Moreover, the coupling is localized on the brane, so that no signature remains from the non-trivial geometry in the bulk.

\section{Short-wavelength solution in the five-dimensional model}

In the short-wavelength limit, the scalar field perturbations can be approximated by $\delta \varphi=\left(a_{0} \sqrt{2 k}\right)^{-1} \exp [-i(k /$ 
$\left.\left.a_{0}\right) t\right]$, and $k / a_{0}$ is a slowly varying parameter $(\dot{\delta} \dot{\varphi}=$ $\left.-i\left(k / a_{0}\right) \delta \varphi\right)$. We write the master equation with $H=0$ (and accordingly, with $n=1)$ :

$$
\ddot{\Sigma}-\frac{\Delta}{a_{0}^{2}} \Sigma-\Sigma^{\prime \prime}=0 .
$$

The brane source term that was proportional to $H$ has disappeared since the jump in $\Sigma^{\prime}$ across the brane is found to be negligible is the limit in which the matter perturbations on the brane behave like a fluid with sound speed $c_{s}^{2}=-1$ (i.e., $\left.\delta T_{0}^{0}=-\delta T_{i}^{i}\right)$. Since $\Sigma^{\prime}$ is odd, this implies $\left[\Sigma^{\prime}\right]_{0}^{2 \pi R}=0$. The most general even solution of Eq. (69) can be written as a Kaluza-Klein expansion (with $p=0,1, \ldots, \infty$ ):

$$
\begin{aligned}
\Sigma= & \sum_{p=-\infty}^{+\infty} \Sigma_{p}=\sum_{p=-\infty}^{+\infty}\left[c_{p} \exp \left(i \nu_{p} t\right)+d_{p} \exp \left(-i \nu_{p} t\right)\right] \\
& \times \cos \left[\omega_{p}(y-\pi R)\right], \\
\nu_{p} \equiv & \left(\frac{k^{2}}{a_{0}^{2}}+\omega_{p}^{2}\right)^{1 / 2},
\end{aligned}
$$

where $\left(c_{p}, d_{p}\right)$ are constants of integration and the $\omega_{p}$ 's are imposed by the continuity of $\Sigma^{\prime}$ :

$$
\omega_{p}=\frac{p}{R}, \quad p=0,1, \ldots, \infty
$$

Inserting the general solution into the integrability condition Eq. (51), which now reduces to

$$
\int_{0}^{2 \pi R} d y \dot{\Sigma}=M_{*}^{-3 \dot{\bar{\varphi}} \delta \varphi}
$$

gives the two constraints

$$
c_{0}=0, \quad d_{0}=\frac{1}{2 \pi R M_{*}^{3}} \frac{i \dot{\bar{\varphi}}}{\sqrt{2 k^{3}}} .
$$

This implies

$$
\Sigma_{p=0}=\frac{1}{2 \pi R M_{*}^{3}} i \frac{a_{0}}{k} \dot{\bar{\varphi}} \delta \varphi
$$

while the constants of integration for $p \geqslant 1$ remain arbitrary. In other words, the zero mode is driven by the scalar field, as would be the case for $\phi_{0}$ in four dimensions, while the heavy Kaluza-Klein modes are independent of the matter on the brane. So, the quantization should be done first for the zero mode and the scalar field together since they only represent one independent degree of freedom, then for each heavy Kaluza-Klein mode separately following the same procedure as for the tensor modes (normalization to the adiabatic vacuum).

Let us focus on the quantization of the zero mode plus the field, since we know that the heavy Kaluza-Klein modes will decouple when $k R / a_{0} \ll 1$. We first must determine whether it is consistent to assume that the metric zero-mode contribution to the perturbed Klein-Gordon equation is very small, and that the field perturbation can be quantized as a free field, while the metric zero mode just follows. In order to discover the answer, we need to compute the contribution to $\left[\Psi_{55}\right]_{0}^{2 \pi R}$ arising from the zero mode only. This is done by integrating Eq. (48), which now simplifies to

$$
\frac{3}{2} \Sigma^{\prime \prime}-\frac{k^{2}}{a_{0}^{2}} \Sigma+\frac{3}{4} \Psi_{55}^{\prime \prime}=M_{*}^{-3} \delta T_{0}^{0} \delta(y) .
$$

Replacing $\Sigma$ by $\Sigma_{p=0}$, and using the constraint $\int_{0}^{2 \pi R} d y \Psi_{55}$ $=0$, we get

$$
\left(\Psi_{55}\right)_{p=0}=\frac{2}{3} \frac{k^{2}}{a_{0}^{2}}\left[(y-\pi R)^{2}-\frac{1}{3}(\pi R)^{2}\right] \Sigma_{p=0} .
$$

By evaluating this relation at $y=0$ we find that the relation between $\phi_{0}$ and $\psi_{0}$ arising from the zero mode is

$$
\psi_{0}-\phi_{0}=\frac{2(k \pi R)^{2}}{9 a_{0}^{2}}\left(\psi_{0}+\phi_{0}\right)
$$

Let us first examine the limit $H R \ll k R / a_{0} \ll 1$ in which we expect to recover the four-dimensional results. Indeed, in this case we find from Eqs. (75) and (78) that

$$
\phi_{0}=\psi_{0}=\frac{1}{4 \pi R M_{*}^{3}} i \frac{a_{0}}{k} \dot{\bar{\varphi}} \delta \varphi=\frac{1}{2 M_{P}^{2}} i \frac{a_{0}}{k} \dot{\bar{\varphi}} \delta \varphi
$$

which is exactly the four-dimensional result of Eq. (57). On the other hand, in the limit $k R / a_{0} \gg 1$ the contribution to $\phi_{0}$ and $\psi_{0}$ arising from the zero mode is

$$
-\phi_{0}=\psi_{0}=\frac{(k \pi R)^{2}}{9 a_{0}^{2}} \frac{1}{2 \pi R M_{*}^{3}} i \frac{a_{0}}{k} \dot{\bar{\varphi}} \delta \varphi .
$$

So, the right-hand side in the perturbed Klein-Gordon equation is of order

$$
\begin{aligned}
\left|\dot{\bar{\varphi}}\left(\dot{\phi}_{0}+3 \dot{\psi}_{0}\right)\right| & =\left(\frac{\sqrt{2} k \pi R}{3 a_{0}}\right)^{2} \frac{\dot{\bar{\varphi}}^{2}}{2 \pi R M_{*}^{3}}|\delta \varphi| \\
& =\left(\frac{2 k \pi R}{3 a_{0}}\right)^{2}|\dot{H} \delta \varphi| \\
& \ll\left(\frac{2 \pi R H k}{3 a_{0}}\right)^{2}|\delta \varphi|,
\end{aligned}
$$

where we used the slow-roll inequality $\epsilon=-\dot{H} / H^{2} \ll 1$. If we remember that $\pi R H<1$ and that the leading terms in the homogeneous perturbed Klein-Gordon equation are of order $\left(k / a_{0}\right)^{2}|\delta \varphi|$, we see that even in this regime, the dynamics of the scalar field is unaffected by that of the metric perturbations. This justifies the assumption that we made in the 
long wavelength regime that the field dynamics is the same as in four-dimensional physics (same vacuum normalization and same evolution).

\section{THE CONSISTENCY RELATION}

Four-dimensional single-field models of inflation predict a consistency relation [17] relating the amplitude of the scalar perturbations, $\mathcal{P}_{S}(k)$, the amplitude of the tensor perturbations, $\mathcal{P}_{T}(k)$, and the tensor spectral index, $n_{T}$ $\equiv d \ln \mathcal{P}_{T}(k) / d \ln k$.

Indeed, in four dimensions since $\mathcal{P}_{T}(k) \propto H^{2}(k), n_{T}$ is given by $n_{T}=d \ln H^{2}(k) / d \ln k=-2 \epsilon$. The four-dimensional consistency relation is

$$
\left.\frac{\mathcal{P}_{T}(k)}{\mathcal{P}_{S}(k)}\right|_{4 D}=16 \epsilon=-8 n_{T} .
$$

In the five-dimensional universe, however, both the amplitude and the tilt of the tensor power spectrum receive corrections which are functions of $(R H)^{2}$.

If we parametrize the $R$-dependent corrections to the power spectrum of tensor modes as

$$
\mathcal{P}_{T}(k)=\frac{2}{\pi^{2}}\left[\frac{H(k)}{M_{P}}\right]^{2} \frac{1}{1-\alpha R^{2} H^{2}(k)}
$$

(recall that our result was $\alpha=2 \pi^{2} / 3$ ), we may compute the spectral index of the tensor modes to be

$$
\begin{aligned}
n_{T} & =\frac{d \ln \mathcal{P}_{T}(k)}{d \ln H^{2}(k)} \frac{d \ln H^{2}(k)}{d \ln k} \\
& =\frac{1}{1-\alpha R^{2} H^{2}} \frac{d \ln H^{2}(k)}{d \ln k} \\
& =-\frac{2 \epsilon}{1-\alpha R^{2} H^{2}},
\end{aligned}
$$

where we have used the fact that the change of the Hubble parameter as a function of scale, $d \ln H^{2}(k) / d \ln k$, is still given by $-2 \epsilon$ since the inflaton field is a brane field and the Hubble rate still satisfies the four-dimensional equation $\dot{H}$ $=-\epsilon H^{2}$.

Using Eqs. (37), (67), and (84), we find

$$
\frac{\mathcal{P}_{T}(k)}{\mathcal{P}_{S}(k)}=\frac{16 \epsilon}{1-\alpha R^{2} H^{2}}=-8 n_{T} .
$$

This is a particularly surprising result: the consistency relation remains unaltered at lowest order in the slow-roll parameters. A similar result has been found in Ref. [19] for a set-up where the bulk on either side of the brane corresponds to anti-de Sitter (AdS) spaces with different cosmological constants. This degeneracy between the usual result in $4 \mathrm{D}$ onefield inflation and in extra-dimensional models will make it more difficult to disentangle the various theoretical possibilities from observations. Our results hold in the case in which only curvature perturbations are generated during the inflationary phase. If isocurvature perturbations are produced, the consistency relation in brane world scenarios is expected to differ from the one obtained here as it happens in the fourdimensional case [20].

Of course this conclusion depends strongly on the particular form of the corrections to the power spectrum of the tensor perturbations, $\left(1-\alpha R^{2} H^{2}\right)^{-1}$, which holds in our five-dimensional example. One can show that this is actually the only possible functional dependence on $H$ such that the consistency relation remains unaltered.

However, our result seems to be quite robust: even considering more than one extra dimension or even a RandallSundrum-like scenario, we show in the next section that the power spectrum of tensor perturbations always gets corrections of the same form, as long as the radius is completely stabilized during inflation.

Of course our calculation of the consistency relation is only to lowest order in slow-roll parameters. In general, one expects the usual four-dimensional corrections to the lowestorder result, corrections from the five-dimensional background equations if $H$ is not constant, and corrections of order $\left(H^{2} R^{2}\right)$ to the scalar perturbations as indicated in Eq. (65).

\section{GENERALIZATION OF THE RESULTS}

We now would like to generalize some of our considerations to the case of more than one extra dimension in the case in which the sizes of the extra dimensions are all equal. If we assume that the compactified geometry of the extra dimensions is stabilized, we can take the background metric in the form of Eq. (1) with $d y^{2} \equiv \delta_{\alpha \beta} d y^{\alpha} d y^{\beta}, \quad \alpha, \beta$ $=1, \ldots, \delta$. Equations (2) and (4) for $\delta$ extra dimensions become

$$
\begin{aligned}
G_{0}{ }^{0} & =\frac{3}{a^{2}}\left(\frac{\dot{a}^{2}}{n^{2}}-\frac{1}{2} \partial_{\alpha} \partial^{\alpha} a^{2}\right) \\
& =M_{*}^{-(2+\delta)} \rho(t) \delta\left(y_{1}\right) \ldots \delta\left(y_{\delta}\right), \\
G_{\alpha}{ }^{0} & =-\frac{3}{a n} \partial_{\alpha}\left(\frac{\dot{a}}{n}\right)=0 .
\end{aligned}
$$

Let us now make the simplifying assumption that because of rotational symmetry in the extra dimensions the scale and lapse functions $a$ and $n$ depend only on the distance from the brane, $r \equiv\left(\Sigma_{\alpha} y_{\alpha}^{2}\right)^{1 / 2}$, and on time, but not on the angular variables. Then, Eq. (86) becomes

$$
\begin{aligned}
G_{0}{ }^{0} & =-\frac{3}{2 a^{2}}\left[\frac{d^{2} a^{2}}{d r^{2}}+\frac{(\delta-1)}{r} \frac{d a^{2}}{d r}-2 K^{2}\right] \\
& =M_{*}^{-(2+\delta)} \rho(t) \frac{1}{S_{\delta} r^{\delta-1}} \delta(r),
\end{aligned}
$$

where $S_{\delta}$ is the surface of a unit-radius sphere in $\delta$ dimensions. The lapse function is given by $n=\dot{a} / K$, where $K$ is 
independent of $r$, so we can choose $K=\dot{a}(\bar{r}) / n(\bar{r})$, evaluated at an arbitrary point $r=\bar{r}$. For $\delta>1$, the solution of Eq. (88) becomes singular at $r=0$, where the brane is located.

To overcome this difficulty, we define a brane with a finite thickness, and impose our boundary conditions at $r=\epsilon$, keeping only the leading terms in the limit $\epsilon \rightarrow 0$. We assume that appropriate density terms within the brane smooth the singularity at the origin. Next, we require compactification conditions that, for simplicity, involve only the variable $r$, and we impose that the values of the scale factor $a$ at $r=\epsilon$ and at $r=2 \pi R-\epsilon$ are equal. Then, the solution of Eq. (88) is

$$
a=a_{\epsilon} \sqrt{1-(\pi R H)^{2} c(r)},
$$

with $c(r)$ given by

$$
c(r)= \begin{cases}\frac{2 r}{\pi R}-\left(\frac{r}{\pi R}\right)^{2} & \text { for } \delta=1, \\ \frac{2 \ln r / \epsilon}{\ln 2 \pi R / \epsilon}-\frac{1}{2}\left(\frac{r}{\pi R}\right)^{2} & \text { for } \delta=2, \\ \frac{4}{\delta}\left[1-\left(\frac{\epsilon}{r}\right)^{\delta-2}\right]-\frac{1}{\delta}\left(\frac{r}{\pi R}\right)^{2} & \text { for } \delta \geqslant 3 .\end{cases}
$$

Here $a_{\epsilon}=a(r=\epsilon)$, and $H=\dot{a}_{\epsilon} /\left(n_{\epsilon} a_{\epsilon}\right)$ is determined to be proportional to $\sqrt{\rho}$ by the jump condition of $d a / d r$. In the case $\delta=1$ we can safely take the limit $\epsilon \rightarrow 0$ and we reproduce Eq. (5).

The correction factor for the tensor perturbations can be obtained following the same argument used in Sec. III. The effective gravitational Planck mass during inflation is

$$
\left.M_{P}^{2}\right|_{I}=M_{*}^{\delta-2} \int d^{\delta} y n^{2}=M_{P}^{2}\left[1-(\pi R H)^{2} C\right]
$$

with

$$
C \equiv \frac{\int_{\epsilon}^{2 \pi R-\epsilon} d r r^{\delta-1} c(r)}{\int_{\epsilon}^{2 \pi R-\epsilon} d r r^{\delta-1}} .
$$

The coefficient $C$ depends on the compactification geometry. In the five-dimensional case studied in Sec. III, we found $C=2 / 3$, while in the simplified case of $\delta$ extra dimensions compactified as explained above, we obtain $C=8 /[\delta(\delta$ $+2)$ ] (for $\delta \geqslant 2$ ).

In conclusion, our result that the tensor perturbations are enhanced by a factor $\left[1-(\pi R H)^{2} C\right]^{-1}$ is a generic consequence of our setup with a stabilized geometry. The factor $C$ depends on the details of the compactification and on the number of extra dimensions, but it is typically a number of order unity. This form of corrections for the tensor perturbations (together with the usual four-dimensional result for the scalar perturbations) have the specific property of preserving the consistency relation, as discussed in Sec. V.

We can also extend our result to the case of nonfactorizable geometries [9]. Let us consider a 5-dimensional setup with two branes at $y=0$ and $y=\pi R$, with vacuum densities equal in magnitude but opposite in sign $\left(V_{0}=\right.$ $-V_{\pi}$ ), and a cosmological constant $\Lambda$ in the bulk. We assume the usual relation between $V_{0}$ and $\Lambda$ to obtain the warped Randall-Sundrum metric in vacuum, and we include a constant energy density $\rho$ on the negative-tension brane. Imposing the condition that the compactification radius is stabilized during the cosmological evolution, we obtain that the scale and lapse functions are given by [21]

$$
a=a_{\pi} \frac{n}{\Omega}, \quad \Omega \equiv e^{-\pi K R},
$$

$$
n^{2}=e^{-2 K|y|}\left[1+\frac{\left(2 \Omega^{2}-1\right) H^{2}}{4 \Omega^{2} K^{2}}\right]+\left(\frac{e^{2 K|y|}}{2}-1\right) \frac{\Omega^{2} H^{2}}{2 K^{2}},
$$

where the 5-dimensional coordinate $y$ is defined in the interval $(-\pi R, \pi R)$. Here $K \equiv \Lambda / V_{\pi}$ is the inverse of the AdS radius, and $H \equiv \dot{a}_{\pi} /\left(n_{\pi} a_{\pi}\right)$ is the Hubble constant on the visible brane located at $y=\pi R$, with $a_{\pi}=a(y=\pi R)$ and $n_{\pi}=\Omega$. The Hubble constant is related to the energy density $\rho$ by the equation

$$
H^{2}=\frac{K \rho}{3 M_{*}^{3}\left(1-\Omega^{2}\right)} .
$$

We can now compute the Planck mass during inflation, which is given by

$$
\begin{aligned}
\left.M_{P}^{2}\right|_{I} & \equiv M_{*}^{3} \int_{-\pi R}^{\pi R} d y n^{2} \\
& =\frac{M_{*}^{3}}{K}\left(1-\Omega^{2}\right)\left[1+\frac{H^{2}}{K^{2}}\left(\frac{3 \Omega^{2}-1}{4 \Omega^{2}}-\frac{\pi R K \Omega^{2}}{1-\Omega^{2}}\right)\right] .
\end{aligned}
$$

Recalling that for the Randall-Sundrum model the Planck mass of the 4-dimensional effective theory is given by $M_{P}^{2}$ $=\left(M_{*}^{3} / K\right)\left(1-\Omega^{2}\right)$, we obtain in the limit of small warp factor $\Omega \ll 1$,

$$
\left.M_{P}^{2}\right|_{I}=M_{P}^{2}\left[1-\left(\frac{x_{1} H}{2 m_{1}}\right)^{2}\right] .
$$

Here $m_{1}=x_{1} K \Omega$ is the mass of the first graviton KaluzaKlein mode (in the limit $H=0$ ), and $x_{1}=3.8$ is the first root of the Bessel function $J_{1}$. Therefore, the correction factor is quadratic in $H$ also in the case of factorizable geometries, and the typical scale is determined by the Kaluza-Klein mass gap. 
We should note that in the case of the Randall-Sundrum model, the energy separation between the Kaluza-Klein graviton mass and the fundamental scale at which the theory becomes strongly interacting is often very small and this is a limitation for the applicability of our result. Uncomputable quantum gravity effects can become important and lead to comparable contributions.

\section{CONCLUSIONS}

In this paper we have initiated the investigation of the effects of transdimensional physics on the spectrum of the cosmological density perturbations generated during a period of primordial inflation taking place on our visible threebrane. We have shown that the size of the transdimensional effects are of order $(H R)^{2}$, where $H$ is the Hubble parameter during inflation and $R$ is the typical size of the extra dimensions (or, more precisely, the inverse of the Kaluza-Klein mass gap at zero temperature). The corrections appear in the power spectrum of the tensor modes. The coefficient of the corrections depends upon the compactification geometry, the number of extra dimensions, and if they are flat or warped. As we have already stressed in the Introduction, our treatment should be unaffected by (unknown) quantum effects which might arise at distances below $M_{*}^{-1}$ as long as the size of extra dimensions is larger than $M_{*}^{-1}$.

Our results may be generalized in different ways. First of all, our setup is the simplest we could imagine: only one extra dimension and inflation taking place on the brane. One can envisage the possibility of putting the inflaton field responsible for inflation in the bulk. In such a case we expect a different form of corrections. In particular, the power spectrum of scalar perturbations should be modified, thus possibly changing the consistency relation.

One can also relax our working assumption of keeping the radii of extra dimensions fixed. In this case there might be significant corrections to the slope of the power spectra since having a dynamical radion field during inflation amounts to changing the Hubble rate during inflation.

In our paper we have also assumed that the energy density $\rho$ on the brane is smaller than about $M_{P}^{2} / R^{2}$, or equivalently, that the Hubble radius is larger than the radii of compactification. Deviations from the standard four-dimensional Friedmann law are present in the opposite regime and large deviations from the standard results for the power spectra of density perturbations should appear.

Finally, we have assumed that deep in the ultraviolet regime, at distances much smaller than the horizon length, the initial vacuum is the traditional Bunch-Davies vacuum containing no initial particles in the spectrum. This is a reasonable assumption at physical momenta $k / a_{0}$ much larger than $R^{-1}$, but still smaller than the fundamental scale $M_{*}$. Of course, for momenta $k / a_{0} \gg M_{*}$, unknown quantum effects may take over and change drastically the properties of the vacuum. This would lead to corrections scaling as powers of $H / M_{*}$ as suggested by the analysis performed in the fourdimensional cases [2]. Nevertheless, whenever $M_{*} R$ is sufficiently large, the computable corrections discussed in this paper dominate over these unknown quantum corrections.

\section{ACKNOWLEDGMENTS}

A.R. would like to thank S. Matarrese, D. Lyth, and D. Wands for several discussions and the Theory Group of CERN where part of this work was done. J.L. acknowledges a visit to the University of Padua during which this project was initiated. The work of E.W.K. was supported in part by the Department of Energy and by NASA (NAG5-10842).

\section{APPENDIX: BACKGROUND AND PERTURBED EINSTEIN EQUATIONS}

We give here the background and perturbed Einstein equations in the Gaussian normal gauge:

$$
\begin{aligned}
G_{0}{ }^{0} & =\frac{3}{n^{2}}\left(\frac{\dot{a}}{a}\right)^{2}-3\left[\frac{a^{\prime \prime}}{a}+\left(\frac{a^{\prime}}{a}\right)^{2}\right]-\frac{6}{n^{2}} \frac{\dot{a}}{a}\left(\frac{\dot{a}}{a} \phi+\dot{\psi}\right)+\frac{2}{a^{2}} \Delta \psi+\frac{2}{n^{2} a^{2}} \frac{\dot{a}}{a} \Delta B+\frac{2}{n^{2}} \frac{\dot{a}}{a} \Delta \dot{E}+3\left(4 \frac{a^{\prime}}{a} \psi^{\prime}+\psi^{\prime \prime}\right)-4 \frac{a^{\prime}}{a} \Delta E^{\prime}-\Delta E^{\prime \prime} \\
& =\delta(y)\left[\left(\frac{\dot{\bar{\varphi}}}{2 n^{2}}+V\right)+\frac{\dot{\bar{\varphi}} \delta \dot{\varphi}-\dot{\bar{\varphi}}^{2} \phi}{n^{2}}+V^{\prime} \delta \varphi\right], \\
G_{0}{ }^{i} & =-a^{-2} \partial_{i}\left\{2 \frac{\dot{a}}{a} \phi+2 \dot{\psi}+\frac{2}{n^{2}}\left[\frac{\ddot{a}}{a}-\left(\frac{\dot{a}}{a}\right)^{2}-\frac{\dot{n}}{n} \frac{\dot{a}}{a}\right] B-\left(\frac{n^{\prime \prime}}{n}+\frac{a^{\prime}}{a} \frac{n^{\prime}}{n}\right) B+\frac{1}{2}\left(\frac{a^{\prime}}{a}-\frac{n^{\prime}}{n}\right) B^{\prime}+\frac{1}{2} B^{\prime \prime}\right\} \\
& =-a^{-2} \delta(y) \partial_{i}\left(\dot{\bar{\varphi}} \delta \varphi-\frac{\dot{\bar{\varphi}}^{2}}{n^{2}} B\right)
\end{aligned}
$$




$$
\begin{aligned}
G_{i}{ }^{0}= & n^{-2} \partial_{i}\left\{2 \frac{\dot{a}}{a} \phi+2 \dot{\psi}+\left[\frac{a^{\prime}}{a} \frac{n^{\prime}}{n}-2\left(\frac{a^{\prime}}{a}\right)^{2}-\frac{a^{\prime \prime}}{a}\right] B+\frac{1}{2}\left(\frac{a^{\prime}}{a}-\frac{n^{\prime}}{n}\right) B^{\prime}+\frac{1}{2} B^{\prime \prime}\right\} \\
G_{j}{ }^{i}= & \left\{\frac{1}{n^{2}}\left[2 \frac{\ddot{a}}{a}+\frac{\dot{a}}{a}\left(\frac{\dot{a}}{a}-2 \frac{\dot{n}}{n}\right)\right]-2 \frac{a^{\prime \prime}}{a}-\frac{a^{\prime}}{a}\left(\frac{a^{\prime}}{a}+2 \frac{n^{\prime}}{n}\right)-\frac{n^{\prime \prime}}{n}-\frac{2}{n^{2}}\left[2 \frac{\ddot{a}}{a}+\left(\frac{\dot{a}}{a}\right)^{2}-2 \frac{\dot{a}}{a} \frac{\dot{n}}{n}\right] \phi-2\left(\frac{a^{\prime}}{a}+\frac{n^{\prime}}{n}\right) \phi^{\prime}-\phi^{\prime \prime}-\frac{2}{n^{2}} \frac{\dot{a}}{a} \dot{\phi}\right. \\
& \left.+2\left(\frac{n^{\prime}}{n}+3 \frac{a^{\prime}}{a}\right) \psi^{\prime}+2 \psi^{\prime \prime}-\frac{2}{n^{2}}\left(3 \frac{\dot{a}}{a}-\frac{\dot{n}}{n}\right) \dot{\psi}-\frac{2}{n^{2}} \ddot{\psi}\right\} \delta_{i j}+a^{-2}\left(\delta_{i j} \Delta-\partial_{i} \partial_{j}\right)\left\{-\phi+\psi+\frac{1}{n^{2}}\left[\left(\frac{\dot{a}}{a}-\frac{\dot{n}}{n}\right) B+\dot{B}\right]\right. \\
& \left.+\frac{a^{2}}{n^{2}}\left[\left(3 \frac{\dot{a}}{a}-\frac{\dot{n}}{n}\right) \dot{E}+\ddot{E}\right]-a^{2}\left[\left(3 \frac{a^{\prime}}{a}+\frac{n^{\prime}}{n}\right) E^{\prime}+E^{\prime \prime}\right]\right\} \\
= & \delta(y)\left(V-\frac{\dot{\varphi^{2}}}{2 n^{2}}+\frac{\dot{\varphi^{2}} \phi-\dot{\dot{\varphi}} \delta \dot{\varphi}}{n^{2}}+V^{\prime} \delta \varphi\right) \delta_{i j} \\
G_{5}{ }^{0}= & \frac{3}{n^{2}}\left[\left(\frac{\dot{a}}{a} \frac{n^{\prime}}{n}-\frac{\dot{a}^{\prime}}{a}\right)(1-2 \phi)+\frac{\dot{a}}{a} \phi^{\prime}+\frac{\dot{a}}{a} \psi^{\prime}+\left(\frac{a^{\prime}}{a}-\frac{n^{\prime}}{n}\right) \dot{\psi}+\dot{\psi}^{\prime}\right]+\frac{1}{n^{2} a^{2}}\left(\frac{n^{\prime}}{n} \Delta B-\frac{1}{2} \Delta B^{\prime}\right) \\
& +\frac{1}{n^{2}}\left[-\Delta \dot{E}^{\prime}+\left(\frac{n^{\prime}}{n}-\frac{a^{\prime}}{a}\right) \Delta \dot{E}-\frac{\dot{a}}{a} \Delta E^{\prime}\right]=0 \\
G_{5}{ }^{i}= & a^{-2} \partial_{i}\left(\phi^{\prime}-2 \psi^{\prime}+\left(\frac{n^{\prime}}{n}-\frac{a^{\prime}}{a}\right) \phi+\frac{1}{n^{2}}\left[\frac{a^{\prime}}{a} \dot{B}-\frac{1}{2} \dot{B}^{\prime}+\frac{1}{2}\left(\frac{\dot{n}}{n}-3 \frac{\dot{a}}{a}\right) B^{\prime}+\left(2 \frac{\dot{a} a^{\prime}}{a^{2}}-2 \frac{\dot{a}^{\prime}}{a}-\frac{\dot{n} a^{\prime}}{n a}+3 \frac{\dot{a} n^{\prime}}{a n}\right) B\right]\right\}=0 .
\end{aligned}
$$

These equations simplify in the de Sitter background defined in Eq. (9):

$$
\begin{aligned}
n^{2} \delta G_{0}{ }^{0}= & -6 H(H \phi+\dot{\psi})+2 \frac{\Delta}{a_{0}^{2}} \psi+2 \frac{H \Delta}{a_{0}^{2} n^{2}} B+2 H \Delta \dot{E}+3\left(4 n^{\prime} n \psi^{\prime}+n^{2} \psi^{\prime \prime}\right)-4 n^{\prime} n \Delta E^{\prime}-n^{2} \Delta E^{\prime \prime} \\
-a^{2} \delta G_{0}^{i}= & \partial_{i}\left(2 H \phi+2 \dot{\psi}-\frac{1}{n^{2}} H^{2} B+\frac{1}{2} B^{\prime \prime}\right) \\
n^{2} \delta G_{j}^{i}= & \left(-6 H^{2} \phi-4 n^{\prime} n \phi^{\prime}-n^{2} \phi^{\prime \prime}-2 H \dot{\phi}+8 n^{\prime} n \psi^{\prime}+2 n^{2} \psi^{\prime \prime}-6 H \dot{\psi}-2 \ddot{\psi}\right) \delta_{i j}+\left(a_{0} n\right)^{-2}\left(\delta_{i j} \Delta-\partial_{i} \partial_{j}\right) \\
& \times\left[-\phi+\psi+n^{-2}(H B+\dot{B})+a_{0}^{2}(3 H \dot{E}+\ddot{E})-a_{0}^{2}\left(4 n^{\prime} n E^{\prime}+n^{2} E^{\prime \prime}\right)\right] \\
n^{2} \delta G_{5}{ }^{0}= & 3\left(H \phi^{\prime}+H \psi^{\prime}+\dot{\psi}^{\prime}\right)+\frac{1}{a_{0}^{2} n^{2}}\left(\frac{n^{\prime}}{n} \Delta B-\frac{1}{2} \Delta B^{\prime}\right)-\Delta \dot{E}^{\prime}-H \Delta E^{\prime} \\
-a^{2} G_{5}^{i}= & \partial_{i}\left[-\phi^{\prime}+2 \psi^{\prime}+\frac{1}{n^{2}}\left(\frac{1}{2} \dot{B}^{\prime}+\frac{3}{2} H B^{\prime}-\frac{n^{\prime}}{n} \dot{B}-3 H \frac{n^{\prime}}{n} B\right)\right] .
\end{aligned}
$$

[1] For a review, see D.H. Lyth and A. Riotto, Phys. Rep. 314, 1 (1999).

[2] J. Martin and R.H. Brandenberger, Phys. Rev. D 63, 123501 (2001); J.C. Niemeyer, ibid. 63, 123502 (2001); A. Kempf, ibid. 63, 083514 (2001); J. Martin and R.H. Brandenberger, astro-ph/0012031; J.C. Niemeyer and R. Parentani, Phys. Rev. D 64, 101301 (2001); A. Kempf and J.C. Niemeyer, ibid. 64,
103501 (2001); A.A. Starobinsky, Pisma Zh. Éksp. Teor. Fiz. 73, 415 (2001) [JETP Lett. 73, 371 (2001)]; R. Easther, B.R. Greene, W.H. Kinney, and G. Shiu, Phys. Rev. D 64, 103502 (2001); L. Hui and W.H. Kinney, ibid. 65, 103507 (2002); M. Lemoine, M. Lubo, J. Martin, and J.P. Uzan, ibid. 65, 023510 (2002); R. Easther, B.R. Greene, W.H. Kinney, and G. Shiu, hep-th/0110226; R. H. Brandenberger, S. E. Joras, and J. Mar- 
tin, Phys. Rev. D (to be published) hep-th/0112122; N. Kaloper, M. Kleban, A.E. Lawrence, and S. Shenker, hep-th/0201158; J. Martin and R.H. Brandenberger, Phys. Rev. D 65, 103514 (2002); R.H. Brandenberger and J. Martin, hep-th/0202142; G. Shiu and I. Wasserman, Phys. Lett. B 536, 1 (2002); H. Danielsson, Phys. Rev. D 66, 023511 (2002); S.F. Hassan and M.S. Sloth, hep-th/0204110; R. Easther, B.R. Greene, W.H. Kinney, and G. Shiu, Phys. Rev. D 66, 023518 (2002); U.H. Danielsson, J. High Energy Phys. 07, 040 (2002); J. C. Niemeyer, R. Parentani, and D. Campo, Phys. Rev. D (to be published), hep-th/0206149.

[3] A signature of trans-Planckian physics which does not depend upon nonlinear unknown quantum effects is the one induced by resonant production of the particle during inflation leaving an imprint in the form of sharp features in the primordial power spectrum. This effect has been discussed in D.J. Chung, E.W. Kolb, A. Riotto, and I.I. Tkachev, Phys. Rev. D 62, 043508 (2000).

[4] N. Arkani-Hamed, S. Dimopoulos, and G.R. Dvali, Phys. Lett. B 429, 263 (1998); I. Antoniadis, N. Arkani-Hamed, S. Dimopoulos, and G.R. Dvali, ibid. 436, 257 (1998).

[5] D. Langlois, R. Maartens, and D. Wands, Phys. Lett. B 489, 259 (2000).

[6] R. Maartens, D. Wands, B.A. Bassett, and I. Heard, Phys. Rev. D 62, 041301 (2000); D. Langlois, R. Maartens, M. Sasaki, and D. Wands, ibid. 63, 084009 (2001).

[7] Notice that $a_{0}(t)$ is the value of the scale factor at $y=0$, not its present value.

[8] P. Kanti, K.A. Olive, and M. Pospelov, Phys. Lett. B 481, 386 (2000); C. Csaki, M. Graesser, L. Randall, and J. Terning, Phys. Rev. D 62, 045015 (2000).

[9] L. Randall and R. Sundrum, Phys. Rev. Lett. 83, 3370 (1999); 83, 4690 (1999).

[10] P. Binetruy, C. Deffayet, and D. Langlois, Nucl. Phys. B565, 269 (2000); P. Binetruy, C. Deffayet, U. Ellwanger, and D. Langlois, Phys. Lett. B 477, 285 (2000).

[11] This equation admits the solution $\omega=0$ for any value of $R H$. In the limit of small $R H$, the solutions of Eq. (22) are $\omega_{p}$ $=p / R$, with $p$ ranging over the non-negative integers. Indeed, in the limit $R H \rightarrow 0$, the space is not warped $(n \rightarrow 1)$ and we recover the usual equally spaced Kaluza-Klein eigenvalues. The solutions for $0<\pi R H<1$ are plotted in Fig. 1 .

[12] See, e.g., A. R. Liddle and D. H. Lyth, Cosmological Inflation and Large-Scale Structure (Cambridge University Press, Cambridge, England, 2000).

[13] Notice that if we defined the effective four-dimensional Planck mass during inflation by integrating the background gravitational action over $y$, we would obtain a different result, $\left.M_{P}^{2}\right|_{I}$ $=M_{P}^{2}\left(1-\frac{1}{3} \pi^{2} R^{2} H^{2}\right)$. However, we are interested here in the effective gravitational coupling of the zero Kaluza-Klein mode of the tensor perturbation, which is properly defined by Eq. (39).

[14] R. Maartens, Phys. Rev. D 62, 084023 (2000); C. van de Bruck, M. Dorca, R. Brandenberger, and A. Lukas, ibid. 62, 123515 (2000); H. Kodama, A. Ishibashi, and O. Seto, ibid. 62, 064022 (2000); S. Mukohyama, ibid. 62, 084015 (2000); Class. Quantum Grav. 17, 4777 (2000); Phys. Rev. D 64, 064006 (2001); D. Langlois, ibid. 62, 126012 (2000); Phys. Rev. Lett. 86, 2212 (2001); A. Neronov and I. Sachs, Phys. Lett. B 513, 173 (2001); C. van de Bruck and M. Dorca, hep-th/0012073; H. Bridgman, K. Malik, and D. Wands, Phys. Rev. D 65, 043502 (2002); K. Koyama and J. Soda, ibid. 62, 123502 (2000); 65, 023514 (2002); V. Bozza, M. Gasperini, and G. Veneziano, Nucl. Phys. B619, 191 (2001); O. Seto and H. Kodama, Phys. Rev. D 63, 123506 (2001); J.D. Barrow and R. Maartens, Phys. Lett. B 532, 153 (2002); A. Riazuelo, F. Vernizzi, D. Steer, and R. Durrer, hep-th/0205220.

[15] Langlois, [14].

[16] Mukohyama, [14].

[17] For a review, see J.E. Lidsey, A.R. Liddle, E.W. Kolb, E.J. Copeland, T. Barreiro, and M. Abney, Rev. Mod. Phys. 69, 373 (1997).

[18] V.F. Mukhanov, H.A. Feldman, and R.H. Brandenberger, Phys. Rep. 215, 203 (1992).

[19] G. Huey and J.E. Lidsey, Phys. Rev. D 66, 043514 (2002).

[20] N. Bartolo, S. Matarrese, and A. Riotto, Phys. Rev. D 64, 083514 (2001); 64, 123504 (2001); D. Wands, N. Bartolo, S. Matarrese, and A. Riotto 66, 043520 (2002).

[21] J. Lesgourgues, S. Pastor, M. Peloso, and L. Sorbo, Phys. Lett. B 489, 411 (2000). 